$63^{\text {ème }}$ Congrès de la SFCO, 03029 (2015)

DOI:10.1051/sfco/20156303029

(C) Owned by the authors, published by EDP Sciences, 2015

\title{
Posters
}

\section{À propos d'un cas de nodule labial atypique}

\author{
Calvo A-S***, Cavelier-Balloy B***, Agbo Godeau S****, Godeau B*****, \\ Lescaille $\mathrm{G}^{* * * *}$, Descroix $\mathrm{V}^{* * * *}$, Goudot $\mathrm{P} * * * *$, Hamma-Kollar $\mathrm{E}^{* * * *}$ \\ * Département de chirurgie Orale, hôpital de la Pitié Salpétrière \\ ** Faculté Paris Diderot, UFR odontologie \\ *** Cabinet d'anatomie pathologique et dermatologique, Paris \\ **** Service de Chirurgie Maxillo faciale Hôpital de la pitié Salpétrière \\ ***** Service de médecine interne 1 Hôpital Henri Mondor
}

Il s'agit d'une récidive de lésion nodulaire de la lèvre supérieure gauche chez un homme de 38 ans sans antécédents particuliers. Cette récidive apparaît un an et demi après l'exérèse de la lésion primitive sur le même site. Il s'agit d'un nodule de $28 \mathrm{~mm} \times 10 \mathrm{~mm}$ de diamètre. Début 2013, son médecin généraliste l'avait adressé à un spécialiste en chirurgie orale pour la prise en charge de l'apparition récente d'un nodule souple, mobile par rapport au plan profond et au plan superficiel de $12 \mathrm{~mm}$ de diamètre. Les tissus de recouvrement étaient normaux et la palpation indolore. Les diagnostics différentiels de kystes salivaires ou encore de lipome ont été évoqués à ce stade. Une exérèse chirurgicale a été réalisée. L'aspect macroscopique de la lésion ne correspondait à aucune de ces formations et l'analyse anatomopathologique réalisé en 2013 a conclu à une amylose nodulaire (tissu positif au Red Congo). Compte tenu du dignostic, un bilan général a été réalisé en médecine interne au CHU Henri Mondor (IRM et écho-doppler cardiaque, TDM thoraco-abdomino-pelvien, biopsie des glandes salivaires accessoires, electrophorèse des urines et sérique) qui a éliminé une amylose systémique. Dans ce contexte, sans autre localisation, cette lésion a été étiquetée comme une amylose orale localisée de la muqueuse labiale, une pathologie rare qui n’a été décrite que chez 40 patients (Folkard et al. 2014).

Devant cette récidive, la question de la prise en charge se pose, tant sur le plan systémique que chirurgical, compte tenu de l'éxérèse complète de la lésion primitive. A ce jour, une IRM cervico faciale a été réalisée et retrouve une masse de la région vestibulaire labiale supérieure droite sans extension osseuse profonde. Elle montre aussi une adénomégalie diffuse avec présence d'adénopathie au niveau du secteur 2A gauche.

L'amylose est un ensemble de maladies systémiques se traduisant par des dépôts de chaines protéiques issues d'immunoglobulines. Il existe de très nombreuses formes d'amyloses, les plus connues étant l'amylose AA (ou secondaire à une réaction inflammatoire) et l'amylose AL (ou primitive, mais pouvant être associée à un myélome). Tous les organes peuvent être atteints exepté le système nerveux central, et les conséquences systémiques graves. La médiane de survie sans traitement efficace des patient atteints d'amylose AL est de l'ordre de 6 à 10 mois avec une survie à 10 ans inférieure à $5 \%$ (Kyle et al. 1983, Lachmann et al. 2006). Le chirurgien oral doit donc orienter le patient dans une structure adequate afin de rechercher une atteinte systémique devant un diagnostic de ce type. Cependant une progression est rare donc la prise en charge est conservatrice (Goodman et al. 2005).

This is an Open Access article distributed under the terms of the Creative Commons Attribution License 4.0, which permits unrestricted use, distribution, and reproduction in any medium, provided the original work is properly cited. 
$63^{\text {ème }}$ Congrès de la SFCO

Dans le cas présent, un second bilan général devra être réalisé affin de définir l’atteinte systémique du patient face à la récidive et donc son pronostic à long terme.

Nom et adresse du conférencier

Anne-Sophie CALVO

Hôpital de la Salpétrière

47, boulevard de l'Hôpital

75651 Paris Cedex 13 (France)

a.sophiecalvo@gmail.com 